\section{CORRELAÇÃO ENTRE IDADE, INTENSIDADE DE SINTOMAS PROSTÁTICOS E ACHADOS ULTRASSONOGRÁFICOS}

\author{
Correlation between age, intensity of prostate symptoms and \\ ultrasonographic findings
}

Correlación entre la edad, la intensidad de síntomas prostáticos
y los hallazgos ultrasonográficos
Artigo Original

\title{
RESUMO
}

Objetivo: Investigar a associação entre a intensidade dos sintomas do trato urinário baixo e os achados ultrassonográficos em pacientes atendidos no ambulatório de urologia de um hospital universitário. Métodos: Estudo observacional e transversal realizado em um hospital universitário no ano de 2013, em que pacientes com diagnóstico clínico e ultrassonográfico (USG) de hiperplasia prostática benigna (HPB) foram avaliados através de entrevista direta, por meio de aplicação de formulário com coleta de dados, como idade, aspectos do laudo ultrassonográfico e da quantificação dos sintomas através do Escore Internacional de Sintomas Prostáticos (IPSS). Aplicaram-se os testes Kruskal Wallis, Correlação de Spearman e ANOVA de uma via, considerando-se estatisticamente significativo $p<0,05$. Resultados: Não houve correlação estatisticamente significativa entre as variáveis volume prostático (VP) e pontuação do IPSS ( $\mathrm{p}=0,2$ ), assim como entre as variáveis pontuação do IPSS e idade $(p=0,19)$. Contudo, houve correlação estatisticamente significativa entre as variáveis idade e VP $(p=0,03$ e rho=0,34). Conclusão: O IPSS não mostrou correlação estatisticamente significativa com o volume prostático estimado pela ultrassonografia, nem com a idade do paciente, fato sugestivo de que outras variáveis podem estar envolvidas no resultado desse escore.

Descritores: Hiperplasia Prostática; Ultrassonografia; Sintomas.

\section{ABSTRACT}

Objective: To investigate the association between the intensity of symptoms of lower urinary tract and the ultrasonographic findings in patients attending the urology outpatient clinic of a university hospital. Methods: Observational and cross-sectional study conducted at a university hospital in 2013, in which patients with clinical and ultrasonographic (USG) diagnosis of benign prostatic hyperplasia (BPH) were evaluated through direct interview, by applying a form that collected data such as age, aspects of the ultrasound report, and the quantification of symptoms, by means of the International Prostate Symptom Score (IPSS). The study applied the Kruskal-Wallis test, Spearman correlation, and oneway ANOVA, considering statistical significance level at $p<0.05$. Results: There was no statistically significant correlation between the variables prostatic volume (PV) and IPSS score ( $p=0.2)$, as well as between the variables IPSS score and age $(p=0.19)$. However, there was a statistically significant correlation between the variables age and $P V(p=0.03$ and rho=0.34). Conclusion: The IPSS showed no statistically significant correlation with the prostate volume estimated by ultrasonography or with the patient's age, a suggestive fact that other variables may be involved in the outcome of this score.

Descriptors: Prostatic Hyperplasia; Ultrasonography; Symptoms.

1) Universidade Federal da Paraíba - UFPB - João Pessoa (PB) - Brasil

2) Hospital Universitário Lauro Wanderley - HULW - Universidade Federal da Paraíba - UFPB - João Pessoa (PB) - Brasil
Recebido em: 26/07/2014 Revisado em: 11/10/2014 Aceito em: 03/12/2014 


\section{RESUMEN}

Objetivo: Investigar la asociación entre la intensidad de los síntomas del tracto urinario inferior y los hallazgos ultrasonográficos de pacientes asistidos en el ambulatorio de urología de un hospital universitario. Métodos: Estudio observacional y transversal realizado en un hospital universitario en el año de 2013 con pacientes con el diagnóstico clínico y ultrasonográfico (USG) de hiperplasia de próstata benigna que fueron evaluados a través de una entrevista directa con la aplicación de un formulario con datos de la edad, los aspectos del informe de la ultrasonografía y de la cuantificación de los sintomas a través de la Puntuación Internacional de Síntomas Prostáticos (IPSS). Se aplicó las pruebas de Kruskal Wallis, Correlación de Spearman y ANOVA de una vía, considerándose estadísticamente significativo el $p<0,05$. Resultados: No hubo correlación estadísticamente significativa entre las variables del volumen prostático (VP) y la puntuación del IPSS $(p=0,2)$ asi como entre las variables puntuación del IPSS y la edad $(p=0,19)$. Sin embargo, hubo correlación estadísticamente significativa entre las variables edad y VP $(p=0,03$ y rho=0,34). Conclusión: El IPSS no mostró correlación estadísticamente significativa con el volumen prostático estimado en la ultrasonografía ni con la edad del paciente, hecho que sugiere que otras variables pueden estar involucradas en el resultado de la puntuación.

Descriptores: Hiperplasia Prostática; Ultrasonografia; Sintomas.

\section{INTRODUÇÃO}

Desde 2009, o Sistema Único de Saúde (SUS) tem uma política específica de promoção à saúde masculina, de modo a favorecer a maior inclusão dos homens nos serviços de saúde. A porta de entrada é a Atenção Básica, que atua sobre as doenças mais prevalentes ${ }^{(1)}$. Contudo, a hiperplasia prostática benigna (HPB), condição extremamente comum, não está adequadamente contemplada na Política Nacional de Atenção Integral à Saúde do Homem ${ }^{(2)}$. A HPB se caracteriza pelo aumento não cancerígeno da próstata. Trata-se de condição patológica relevante e a segunda causa de intervenção cirúrgica em homens idosos. Idade, hereditariedade e função testicular são fatores de risco $^{(3)}$.

Os sintomas, inicialmente, são discretos, comprometendo progressivamente a qualidade de vida, sendo classificados como obstrutivos e irritativos. Os primeiros são dificuldades para iniciar o jato urinário (hesitância), dificuldade e esforço miccional, jato urinário fraco e fino, e presença de resíduo urinário pós-miccional, devido ao esvaziamento incompleto da bexiga. Os segundos são a dificuldade ou incapacidade de controlar a urina, acordar numerosas vezes à noite para urinar (noctúria) e urinar várias vezes por dia (poliúria) $^{(4)}$. Essas alterações resultam basicamente de três mecanismos patológicos: obstrução infravesical da próstata, reação do músculo da bexiga (detrusor) à obstrução e a estímulos neurais gerados pela próstata ${ }^{(3)}$.

A rigor, homens com mais de quarenta anos ou com sintomas urinários deveriam ser avaliados pelo profissional de saúde quanto à possibilidade de HPB. Primeiramente, o paciente deve responder ao Escore Internacional de Sintomas Prostáticos (IPSS) ${ }^{(5)}$, que estratifica os sintomas através de um questionário composto de perguntas que enfocam a frequência de sete dos principais sintomas associados à doença. Depois, realiza-se o exame de toque retal, que contribui na avaliação da possibilidade de câncer de próstata e estima o volume da glândula, fundamental para a escolha terapêutica ${ }^{(1)}$. Em seguida, o médico deve solicitar exames complementares, como dosagem do antígeno prostático específico (PSA), função renal e ultrassonografia ${ }^{(1)}$. Esta última estima com maior acurácia o volume da próstata e permite avaliar a medida do resíduo pós-miccional, que acusa comprometimento do músculo detrusor quando está acima de $150 \mathrm{ml}$ e lauda o volume prostático como normal $(20 \mathrm{~g})$ ou aumentado (maior que $20 \mathrm{~g})^{(6)}$.

Quando a sintomatologia é de moderada a grave, a qualidade de vida dos homens acometidos por HPB fica comprometida, pois há impacto na saúde física e mental, com limitação do desempenho em atividades diárias, alteração do padrão do sono e deterioração da função sexual ${ }^{(7)}$. Portanto, tratando-se de uma doença muito prevalente e com o diagnóstico relativamente simples ${ }^{(1)}$, é fundamental para os profissionais que atuam nas Unidades Básicas de Saúde (UBS) reconhecer quais são os pacientes portadores de HPB que precisam ou não da avaliação de um especialista em Urologia e, assim, otimizar custos e tempo. Um exemplo vem da Atenção Primária na Espanha, em que homens com mais de 50 anos são rastreados para HPB com protocolo especifico ${ }^{(8)}$. No Brasil, há carência de protocolos que facilitem o rastreio e a intervenção em nível primário, o que contribuiria para o correto encaminhamento ao urologista.

Diante da propedêutica investigativa para HPB, que envolve a anamnese com aplicação do IPSS, o exame físico com toque retal, e a ultrassonografia para aferição do volume prostático e do resíduo pós-miccional, é importante conhecer se há correlação significativa entre esses parâmetros, a fim de reduzir gastos com solicitações de exames. Baseado no exposto, o presente estudo objetivou investigar a associação entre a intensidade dos sintomas do trato urinário baixo e os achados ultrassonográficos em pacientes atendidos no ambulatório de urologia de um hospital universitário. 


\section{MÉTODOS}

O presente estudo seguiu um modelo observacional e transversal. A amostra, selecionada de modo não probabilístico e por conveniência, consistiu de pacientes com diagnóstico estabelecido de hiperplasia prostática benigna, atendidos no ambulatório de Urologia do Hospital Universitário Lauro Wanderley (HULW), em João Pessoa, Paraíba, no período de maio a agosto de 2013.

Selecionaram-se para o estudo os pacientes que possuíam ultrassonografia abdominal ou transretal e que aceitaram participar do estudo, através da assinatura do Termo de Consentimento Livre e Esclarecido. Excluíram-se do estudo os pacientes que se recusaram a assinar o Termo de Consentimento Livre e Esclarecido, que passaram por cirurgia prostática ou vesical prévia, portadores de estenose uretral ou disfunção vesical neurogênica, com uso de alfabloqueador ou de inibidor da 5-alfa-redutase, com exame ultrassonográfico realizado há mais de seis meses antes da coleta de dados ou ausência de avaliação do peso prostático por ultrassonografia.

Utilizou-se a técnica de entrevista direta com o paciente, por meio da aplicação de um formulário e do questionário do Escore Internacional de Sintomas Prostáticos (IPSS), para a coleta de dados. Para calibração do entrevistador, um acadêmico de Medicina que já tinha cursado a disciplina de Urologia, capacitado por professor doutor em Urologia para coleta de dados, aplicou um pré-teste em cinco pacientes para observar problemas de operacionalização e corrigir possíveis erros metodológicos. No formulário, registraram-se a variável idade e o laudo ultrassonográfico emitido pelo radiologista responsável, havendo permissão da instituição e do paciente, por meio da assinatura dos termos de anuência e de consentimento, para registro das informações. A quantificação dos sintomas foi realizada por meio do IPSS (Quadro 1).

A partir dos dados obtidos, classificaram-se os pacientes em três grupos, segundo a pontuação obtida: sintomas leves ( 0 a 7 pontos), sintomas moderados ( 8 a 19 pontos) e sintomas severos (20 a 35 pontos).

Para análise estatística, utilizou-se o software SPSS 19.0 para Windows, por meio dos testes Shapiro-Wilks para avaliar a normalidade das variáveis ordinais volume prostático e idade, Kruskal Wallis para avaliação da correlação entre volume prostático a pontuação do IPSS (agrupado em sintomas leves, moderados e severos), e Correlação de Spearman para avaliar a correlação entre idade e volume prostático. Utilizou-se a ANOVA de uma via para avaliar a correlação entre grau de IPSS e idade.

O presente estudo obteve aprovação do Comitê de Ética em Pesquisa do HULW através do número de aprovação 261.262.

Quadro 1 - Escore Internacional de Sintomas Prostáticos (IPSS).

\begin{tabular}{|l|l|l|l|l|l|l|}
\hline & 0 & Até 5 & Até 14 & $\mathbf{1 5}$ & Mais de 15 & Quase sempre \\
\hline $\begin{array}{l}\text { No último mês, quantas vezes ficou com a sensação de } \\
\text { não esvaziar completamente a bexiga? }\end{array}$ & & & & & \\
\hline $\begin{array}{l}\text { No último mês, quantas vezes teve de urinar novamente } \\
\text { menos de 2 horas após de ter urinado? }\end{array}$ & & & & & & \\
\hline $\begin{array}{l}\text { No último mês, quantas vezes observou que, ao urinar, } \\
\text { parou e recomeçou várias vezes? }\end{array}$ & & & & & & \\
\hline $\begin{array}{l}\text { No último mês, quantas vezes observou que foi difícil } \\
\text { conter a urina? }\end{array}$ & & & & & & \\
\hline $\begin{array}{l}\text { No último mês, quantas vezes observou que o jato } \\
\text { urinário estava fraco? }\end{array}$ & & & & & \\
\hline $\begin{array}{l}\text { No último mês, quantas vezes teve de fazer força para } \\
\text { começar a urinar? }\end{array}$ & & & & & \\
\hline $\begin{array}{l}\text { No último mês, quantas vezes, em média, teve de se } \\
\text { levantar à noite para urinar? }\end{array}$ & & & & & \\
\hline Escore total & & & & & \\
\hline
\end{tabular}

Fonte: Emberton M, Cornel EB, Bassi PF, Fourcade RO, Gómez JMF, Castro R. Benign prostatic hyperplasia as a progressive disease: a guide to the risk factors and options for medical management. International Journal of Clinical Practice. 2008;62(7):1076-1086. 


\section{RESULTADOS}

A amostra constituiu de 42 pacientes, com idade média de 59,5 anos $\pm 10,8$ e volume prostático $52 \mathrm{ml} / \mathrm{cm}^{3}$ $\pm 22,3$. Houve predominância de pacientes com sintomas moderados, seguido dos que apresentaram sintomas severos e leves (Tabela I).

Tabela I - Severidade dos sintomas de acordo com o Escore Internacional de Sintomas Prostáticos (IPSS). João PessoaPB, 2013.

\begin{tabular}{lcc}
\hline IPSS & Numero de Pacientes & Frequência \\
\hline Leve & 11 & $26,2 \%$ \\
Moderado & 16 & $38,1 \%$ \\
Severo & 15 & $35,7 \%$ \\
\hline
\end{tabular}

Não houve correlação estatisticamente significativa entre as variáveis volume prostático e pontuação do IPSS $(\mathrm{p}=0,2)$, assim como entre as variáveis pontuação do IPSS e idade $(\mathrm{p}=0,19)$.

Contudo, houve correlação estatisticamente significativa entre as variáveis idade e volume prostático $(p=0,03$ e rho $=0,34)($ Tabela II).

Tabela II - Correlação entre volume prostático, Escore Internacional de Sintomas Prostáticos (IPSS) e idade. João Pessoa-PB, 2013.

\begin{tabular}{ll}
\hline Correlações & Valor de p \\
\hline Volume Prostático X IPSS & 0,2 \\
IPSS X Idade & 0,19 \\
Idade X Volume prostático & 0,03 \\
\hline
\end{tabular}

\section{DISCUSSÃO}

No presente estudo, a HPB (hiperplasia prostática benigna) acometeu homens com idade média de 59,5 anos e com volume prostático médio de $52 \mathrm{~g}$. Outros estudos apontam para idades médias de $62,5 \operatorname{anos}^{(9)}$ e $53,8 \operatorname{anos}^{(10)}$. Já é consenso que a doença apresenta íntima relação com a idade, podendo acometer até $90 \%$ dos pacientes com mais de 80 anos $^{(11)}$. Um estudo estimou um volume prostático médio de 43,8 $\mathrm{g}$ em pacientes acometidos por tumores não cancerosos da próstata, grupo em que se inclui a $\mathrm{HPB}^{(12)}$. Outro trabalho, realizado com 155 pacientes portadores de HPB, demonstrou volume prostático médio de $42,9 \mathrm{~g}^{(13)}$.

A hiperplasia prostática benigna é uma doença complexa e de caráter progressivo em homens. Comumente, é associada a sintomas do trato urinário inferior, podendo resultar em complicações como retenção urinária aguda e necessidade de cirurgia, comprometendo a qualidade de vida dos homens ${ }^{(4)}$.

Portanto, há uma íntima relação entre idade avançada e aumento do volume prostático, o que sugere que as pessoas com 60 anos e mais possuem maior risco para o desenvolvimento de HPB. Nessa perspectiva, os serviços de saúde, especialmente os da atenção primária, devem desenvolver ações de prevenção que possibilitem a identificação precoce dos homens com maior risco para desenvolvimento do HPB, tendo como objetivos otimizar a abordagem terapêutica ${ }^{(5)}$ e minimizar os danos à saúde do homem.

No presente estudo, a maioria $(38,1 \%)$ dos pacientes investigados teve sintomas quantificados pelo IPSS como moderados, seguidos por severos e leves. Um estudo realizado em Santa Catarina com 155 pacientes portadores de HPB demonstrou predominância de sintomas moderados, com média de 11,9 pontos ${ }^{(13)}$, o que corrobora com o resultado da atual investigação. A predominância de sintomas moderados e severos sugere que os homens com HPB estão sendo diagnosticados quando a doença já está avançada, o que dificulta a abordagem terapêutica e aumenta os gastos na saúde, com danos, às vezes, irreversíveis à qualidade de vida do homem ${ }^{(13)}$.

Inicialmente composta por uma tríade - aumento volumétrico da próstata, sintomas do trato urinário inferior e obstrução infravesical ${ }^{(14)}-$, o crescimento prostático intravesical está diretamente envolvido na determinação de sintomas urinários da $\mathrm{HPB}^{(15)}$. Isso é explicado porque a próstata crescida passa a funcionar como uma válvula obstrutiva, impedindo o esvaziamento da bexiga. Esse mesmo crescimento glandular compromete a capacidade volumétrica da bexiga, desencadeando o surgimento de sintomas como poliaciúria, noctúria e urgência miccional, que comprometem a qualidade de vida dos pacientes ${ }^{(16,17)}$. Essa condição é agravada, muitas vezes, pelo processo de envelhecimento e pela presença de outras comorbidades, pois quanto maior a idade, maior o risco de desenvolver $\mathrm{HPB}^{(15)}$.

Sabe-se que uma maneira efetiva de combater os sintomas da HPB é a prevenção, por meio da abordagem dos fatores de risco da doença ${ }^{(18)}$. Embora os fatores de risco não modificáveis, como idade, genética e geografia, desempenhem papéis importantes na etiologia da HBP, dados recentes revelaram que fatores de risco modificáveis estão associados com a prevenção da doença e alívio dos sintomas, incluindo obesidade, diabetes, dieta, atividade física e inflamação ${ }^{(18)}$. Estudos publicados na Coreia do Sul sugeriram que a perda de peso e a atividade física regular poderiam ser úteis para o alívio sintomático da $\mathrm{HPB}^{(19,20)}$.

A significativa diferença na expectativa de vida entre homens e mulheres sugere que os primeiros são um grupo 
mais vulnerável, necessitando de programas de promoção da saúde direcionados, que atuem nos fatores de risco modificáveis, a fim de envolver o homem com a sua saúde ou gestão de doença ${ }^{(21)}$. Portanto, as evidências do presente estudo sugerem que a abordagem do paciente com sintomas de trato urinário baixo é multifatorial, tendo a Atenção Primária um papel fundamental na prevenção e detecção precoce da HPB, essenciais para a garantia de qualidade de vida ao homem.

$\mathrm{O}$ presente estudo demonstrou que nem o volume prostático nem a idade têm relação estatisticamente significativa com a pontuação do IPSS. Em um trabalho realizado na Suécia, concluiu-se que não há correlação estatisticamente significativa entre a pontuação do IPSS e o volume prostático, nem entre a pontuação do IPSS e a idade $^{(22)}$. Um estudo recente sugeriu que aumentos isolados na pontuação do IPSS não devem ser usados como preditor de HPB $^{(14)}$. Outros trabalhos ${ }^{(13,15,21)}$ não encontraram correlação significativa entre pontuação do IPSS e idade, nem entre pontuação do IPSS e volume prostático - resultados que também foram demonstrados pelo presente estudo.

Durante a consulta, aspectos do IPSS e da estimativa do volume prostático precisam ser aferidos, inclusive por meio da ultrassonografia, que é um exame de baixo custo e com disponibilidade crescente ${ }^{(23)}$ nos serviços públicos de saúde. Contudo, foi demonstrado que a ultrassonografia pode superestimar o tamanho da próstata em até $30 \%$, sendo a via transretal melhor definidora de tamanho, entretanto, seu caráter invasivo limita sua utilização rotineira ${ }^{(24)}$.

Sendo assim, não basta analisar quantitativamente os parâmetros de HPB. É preciso associá-los aos fatores de risco, que podem ser prevenidos ou atenuados pela promoção da saúde ao homem através da Atenção Primária, o que permite identificar com mais acurácia quais pacientes necessitarão de uma avaliação especializada, e até mesmo de um tratamento mais agressivo, por meio de abordagem cirúrgica $^{(21)}$.

Apesar das limitações decorrentes da pequena amostra de pacientes analisados e da subjetividade da quantificação dos sintomas, própria do IPSS, as evidências do presente trabalho sugerem que a simples aplicação do questionário em homens com suspeita de HPB não é suficiente para sugerir o diagnóstico, devendo o paciente ser conduzido para uma entrevista clínica mais abrangente, que contemple a singularidade de cada sintoma, bem como seus impactos na qualidade de vida do homem.

\section{CONCLUSÃO}

No presente estudo, o Escore Internacional de Sintomas Prostáticos (IPSS) não mostrou correlação estatisticamente significativa com o volume prostático estimado pela ultrassonografia, nem com a idade dos pacientes avaliados.

Sugere-se que outras variáveis de caráter individual, além do simples aumento do volume prostático, possam estar envolvidas no desenvolvimento e severidade dos sintomas, e que a abordagem desses pacientes seja completa e integral, a fim de evitar custos desnecessários, otimizar o tratamento e minimizar o impacto da doença na saúde do homem.

\section{REFERÊNCIAS}

1. Bastos PSH, Daniee AJ, Alcântara BJR. Disfunção erétil: da medicalização à integralidade do cuidado na Atenção Básica. BIS, Bol Inst Saúde. 2012;14(1):1019.

2. Ministério da Saúde (BR), Secretaria de Atenção à Saúde, Departamento de Ações Programáticas Estratégicas. Política Nacional de Atenção Integral à Saúde do Homem, Princípios e Diretrizes. Brasília: Ministério da Saúde; 2009.

3. Silva WB. Explorando o conhecimento dos usuários de duas unidades de saúde da família sobre a hiperplasia benigna prostática [trabalho de conclusão de curso]. Campina Grande: Universidade Estadual da Paraíba, Graduação em Enfermagem, Centro de Ciências Biológicas e da Saúde; 2013.

4. Almeida CJ. A experiência bem-sucedida do Centro de Referência da Saúde do Homem no tratamento da hiperplasia benigna da próstata. BIS, Bol Inst Saúde. 2012;14(1):119-24.

5. Emberton M, Cornel EB, Bassi PF, Fourcade RO, Gómez JMF, Castro R. Benign prostatic hyperplasia as a progressive disease: a guide to the risk factors and options for medical management. Int J Clin Pract. 2008;62(7):1076-86.

6. Fernández JC, Olmo JMC, Fernández-Pro A, Martín JA, Bermúdez FJB, Pulido EM, et al. Criterios de derivación en hiperplasia benigna de próstata para atención primaria. Actas Urol Esp. 2010;34(1):24-34.

7. Roehrborn CG, McConnell JD. Etiology, pathophysiology, epidemiology and natural history of benign prostatic hyperplasia. $9^{\text {th }}$ ed. Philadelphia: WB Saunders; 2009.

8. Strope SA, Yang L, Nepple KG, Adriole GL, Owens PL. Population based comparative e effectiveness of transurethral resection of the prostate and laser therapy for benign prostatic hyperplasia. J Urol. 2012;187(4):1341-5. 
9. Schenk JM, Hunter-Merrill R, Y Zheng, et al. Should modest elevations in prostate-specific antigen, international prostate symptom score, or their rates of increase over time be used as surrogate measures of incident benign prostatic hyperplasia? Am J Epidemiol. 2013;178(5):741-51.

10. Byun HK, Sung YH, Kim W, Jung JH, Song JM, Chung HC. Relationships between prostate-specific antigen, prostate volume, and components of metabolic syndrome in healthy korean men. Korean $\mathrm{J}$ Urol.2012;53(11):774-8.

11. Averbeck MA, Blaya R, Seben RR, Lima NG, Denardin D, Fornari A, et al. Diagnóstico e tratamento da hiperplasia benigna da próstata. Rev AMRIGS. 2010;54(4):471-7.

12. Santos VCT, Milito MA, Marchiori E. Current role of transrectal ultrasonography in the early detection of prostate cancer. Radiol Bras. 2006;39(3):185-92.

13. Bellucci CHS. Associação entre sintomas e achados ultrassonográficos na hiperplasia prostática benigna [trabalho de conclusão de curso]. Florianópolis: Universidade Federal de Santa Catarina; 2005.

14. Schenk JM, Hunter-Merrill R, Zheng Y, Etzioni R, Gulati R, Tangen C, et al. Should Modest elevations in prostate-specific antigen, international prostate symptom score, or their rates of increase over time be used as surrogate measures of incident benign prostatic hyperplasia? Am J Epidemiol. 2013;178(5):741-51.

15. Udeh EI, Ozoemena Ofn, Ogwuche E. The relationship between prostate volume and international prostate symptom score in Africans with benign prostatic hyperplasia.Niger J Med. 2013;21(3):290-5.

16. Agrawal CS, Chalise PR, Bhandari BB. Correlation of prostate volume with international prostate symptom score and quality of life in men with benign prostatic hyperplasia. Nepal Med Coll J. 2008;10(2):104-7.

17. Yoo TK, Cho HJ. Benign prostatic hyperplasia: from bench to clinic. Korean J Urol. 2012; 53(3):139-48.

18. Patel ND, Parsons JK. Epidemiology and etiology of benign prostatic hyperplasia and bladder outlet obstruction. Indian J Urol. 2014;30(2):170-6.
19. Kim JM, Song PH, Kim HT, Moon KH. Effect of obesity on prostate-specific antigen, prostate volume, and international prostate symptom score in patients with benign prostatic hyperplasia. Korean J Urol. 2011;52(6):401-5.

20. Abdollah F, Briganti A, Suardi N, Castiglione F, Gallina A, Capitanio U. Metabolic Syndrome and benign prostatic hyperplasia: evidence of a potential relationship, hypothesized etiology, and prevention. Korean J Urol. 2011;52(8):507-16.

21. Bottorff JL, Seaton CL, Johnson ST, Caperchione CM, Oliffe JL, More K, Tillotson SM. An Updated review of interventions that include promotion of physical activity for adult men. Sports Med. 2015;45(6):775800 .

22. Vesely S, Knutson T, Damber JE, Dicuio M, Dahlstrand C. Relationship between age, prostate volume, prostatespecific antigen, symptom score and uroflowmetry in men with lower urinary tract symptoms. Scand J Urol Nephrol. 2003;37(4):322-8.

23. Presti Jr JC, Kane CJ, Shinohara K, Carroll PR. Neoplasia da glândula próstata. In: Tanagho EA, McAninch JW. Urologia Geral de Smith. $17^{\mathrm{a}}$ ed. Porto Alegre: AMGH; 2010. p.348-74.

24. Nardi AC, Nardozza Júnior A, Fonseca CEC, Truzzi JC, Flios LAS, Sadi MV. Urologia Brasil. São Paulo: Planmark; 2013.

\section{Endereço do primeiro autor:}

Arlindo Monteiro de Carvalho Júnior

Centro de Ciências Médicas - Universidade Federal da

Paraíba

Campus I, Jardim Universitário, S/N

Bairro: Castelo Branco

CEP: 58051-900 - João Pessoa - PB - Brasil

E-mail: amcarvalhojr@yahoo.com.br

\section{Endereço para correspondência:}

João Victor Costa Barreto Brígido

Centro de Ciências Médicas - Universidade Federal da Paraíba

Campus I, Jardim Universitário, S/N

Bairro: Castelo Branco

CEP: 58051-900 - João Pessoa - PB - Brasil

E-mail: jvbrigido@gmail.com 